\title{
Associations between and development of welfare indicators in organic layers
}

\author{
L. K. Hinrichsen ${ }^{1}$, A. B. Riber ${ }^{1 \dagger}$ and R. Labouriau ${ }^{2}$ \\ ${ }^{1}$ Department of Animal Science, Faculty of Science and Technology, Aarhus University, Blichers Allé 20, PO Box 50, DK-8830 Tjele, Denmark; ${ }^{2}$ Department of \\ Mathematics, Faculty of Science and Technology, Aarhus University, Ny Munkegade 118, DK-8000 Aarhus, Denmark
}

(Received 1 June 2015; Accepted 9 December 2015; First published online 12 January 2016)

The retail market share of organic eggs in Denmark is high, and the consumers expect high animal welfare standards in the organic production. Documentation of animal welfare is important, however, knowledge about the associations between animal-based welfare indicators is limited. The aims of the study were to investigate the associations between selected welfare indicators at two ages (peak and end of lay), and to examine the development with age of the chosen welfare indicators. The chosen welfare indicators were Ascaridia galli (roundworm) infection, Heterakis sp. (caecal worm) infection, keel bone damages, back feathering, body feathering, foot damages, comb colour and wounds on the body. An observational study with 12 organic egg farms was conducted in 2012 and 2013 with a total of 214 hens assessed individually at the peak and the end of lay. Insufficient data were obtained on helminth infection at the peak of lay. At the end of lay, all helminth infected hens were positive for A. galli, and only three of them had in addition a Heterakis sp. infection. Foot damages, pale combs and wounds on the body occurred at frequencies $<5 \%$ and were therefore, together with the prevalence of Heterakis $s p$. infection, left out of the analysis of associations. A graphical model was used to analyse the associations between the remaining clinical welfare indicators, A. galli infection, housing systems and age of the hens at end of lay. A. galli infection was only directly associated with back feathering at end of lay $(\mathbb{P}=0.011)$ with an increased incidence of $\mathrm{A}$. galli infection in hens with good back feathering. Between the two visits, the prevalence of hens with keel bone damages increased $(\mathrm{P}<0.001)$, and the plumage condition deteriorated $(\mathrm{P}<0.001$ ), whereas the number of hens with plantar abscess $(\mathbb{P}=0.037)$ and pale combs $(\mathbb{P}=0.020)$ decreased. No significant differences were found for other foot damages or for skin damage. In conclusion, back feathering at end of lay provided information about a possible helminth infection, but this is not a useful indicator in daily on-farm management. In addition, evidence was found that the deterioration of the plumage condition with age was not only due to accumulation of damage over time.

Keywords: Ascaridia galli, laying hen, organic egg production, welfare indicator

\section{Implications}

Management towards improved animal welfare is important, and tools aiding the producers in documenting and predicting welfare problems, such as helminth infections, are valuable. Levels of both plumage and keel bone damages at the peak of lay were found to predict the level at the end of lay. Hens with good back feathering at the end of lay were more likely to have roundworms at the end of lay. The latter association provides information on the status at the end of lay and is therefore not useful in daily on-farm management practices of helminth infection.

\footnotetext{
${ }^{\dagger}$ Email: anja.riber@anis.au.dk
}

\section{Introduction}

Organic egg production in Denmark has increased by a factor 1.4 in the last decade. In 2013,18\% of the eggs delivered at the packing facilities in Denmark were organic (Anonymous, 2015). The motivations of the consumers for buying organic products are human health, environmental concerns and animal welfare (Wier et al., 2008). Thus, the consumers expect higher standards of animal welfare in the organic production compared with conventional production. Management towards improved animal welfare is therefore important, and tools aiding the producers in documenting the level of animal welfare are valuable. One such tool is animal welfare assessment performed using methods like the welfare quality protocol (Welfare Quality, 2009) and the 
animal needs index (Bartussek, 2001). Alternatively, one or a few welfare indicators may be assessed depending on the purpose of the assessment. The welfare indicators may be resource- or animal-based. Resource-based indicators are indirect indicators of the welfare of hens collected by examining the physical environment, whereas animal-based welfare indicators are direct measures collected by examining the response of the hen to the environment (Barnett and Hemsworth, 2009). Several animal-based welfare indicators have been found to be valid, reliable and feasible for on-farm assessment (Arnould et al., 2009). Among these are wounds, poor plumage condition, foot and bone damage (including keel bone damage), which all indicate welfare problems that potentially may be present in the organic egg production (Bestman and Wagenaar, 2014). Attention has just recently been directed to keel bone damages. They seem to be more likely to occur in hens with weaker bones (Fleming et al., 2004). Keel bone damage is a term covering both keel bone deviations and keel bone fractures. Long-term pressure on the keel bone, especially on poorly designed perches, is one identified factor causing keel bone deviations (Tauson and Abrahamsson, 1994; Pickel et al., 2011), whereas keel bone fractures are likely to be caused by collision with housing equipment or other hens (Freire et al., 2003; Sandilands et al., 2009; Stratmann et al., 2015).

Another welfare problem that has received increasing attention in recent years by the poultry sector and producers in Denmark is helminth infections (Hinrichsen et al., 2015). In the organic system, hens are kept in floor (single-tiered) or aviary (multi-tiered) systems, where 1/3 of the indoor area has to be solid and littered, with a maximum stocking density of six hens per square metre and where access to an outdoor area of four square metre per hen is required (Anonymous, 2008). Access to litter and an outdoor area are both potential sources of helminth infections, although litter seems to be of minor importance compared with outdoor access (Heckendorn et al., 2009; Maurer et al., 2009). Compared with barn systems and conventional cages, the prevalence of helminth infections is higher in organic systems (Permin et al., 1999), and the most dominating helminth species are Ascaridia galli (roundworm) and Heterakis sp. (caecal worm; Permin et al., 1999; Sherwin et al., 2013). Clinical welfare problems often depend on the age of the hens. For example, the prevalence of helminth infections increases with increasing age of the hens (Pennycott and Steel, 2001). Furthermore, the prevalence of keel bone damages and plumage damage increases with increased age of the hens (Nicol et al., 2006; Drake et al., 2010; Richards et al., 2012).

Knowledge about the associations between the welfare problems related to helminth infection, plumage condition, keel bone damages, skin, comb colour and feet is limited, and age of the hens is likely to affect the level of the welfare problems. If associations exist, time spent, for example by producers, on assessment of welfare may potentially be reduced. Therefore, a study collecting data on helminth infection and clinical welfare indicators at two ages (peak and end of lay) was conducted. The aim of this study was twofold. The first aim was to investigate the possible associations between selected welfare indicators at two ages (peak and end of lay). The second aim was to examine the development with age of the chosen welfare indicators. The chosen welfare indicators were $A$. galli (roundworm) infection, Heterakis sp. (caecal worm) infection, keel bone damages, back feathering, body feathering, foot damages, comb colour and wounds on the body. The hypothesis of this study was that helminth infection (A. galli and Heterakis sp.) diagnosed at the end of lay was associated with welfare indicators at the peak of lay and end of lay. The ability to predict will be valuable to producers, especially if the prediction is based on a low-cost method.

\section{Material and methods}

A study including 15 Danish commercial organic egg farms was conducted from August 2012 to December 2013. The producers were recruited based on voluntary participation by approaching all organic egg-producers registered in the Danish Central Husbandry Register by letter and phone calls. All the producers that expressed interest in the project were included in the study under the condition that the hens were 32 weeks of age no later than by February 2013 in order to finalise data collection during 2013. Each producer participated with one flock. Three flocks were excluded from the study due to incomplete data collection. Thus, the data presented originate from 12 flocks (Table 1). The flock size was 3000 hens, with the exception of one flock that consisted of 2140 hens. Each flock was visited twice; the first visit was between 30 and 38 weeks of age, named peak of lay, and the second visit was in the week before culling (from 62 to 77 weeks of age), named end of lay. The housing system, hybrid, age at the visits and number of included hens per farm are presented in Table 1. During the peak of lay visit, 100 randomly selected hens from each flock were tagged with brightly coloured numbered plastic leg bands and clinically examined as described below. Before being released back into the flock, they were placed in a cardboard box for $\sim 30 \mathrm{~min}$. Any faecal samples deposited in the box was collected and frozen for later examination of parasitological content.

Of the original 100 tagged hens per flock $11 \%$ to $21 \%$ was relocated at the end of lay visit, resulting in a total of 214 hens (Table 1). The relocated tagged hens underwent a second clinical examination and were then killed by neck dislocation and stored at $-18^{\circ} \mathrm{C}$ until later examination. Each tagged hen was given a unique identification number based on the numbered band and farm of origin.

The clinical examination was performed according to the scoring protocol developed by participants from eight European countries in the Core Organic project Healthy Hens (Table 2; CORE organic HealthyHens project, 2014). The protocol contains only animal-based welfare indicators and they are closely related to those used in the Welfare Quality protocol. The clinical examination was performed by the 
Table 1 Description of the 12 commercial organic layer flocks according to housing system, presence of veranda, hybrid, age at the two visits and the number of hens per flock included in the present study

\begin{tabular}{|c|c|c|c|c|c|}
\hline Housing system & Veranda & Hybrid & Age at the peak of lay visit & Age at the end of lay visit & Hens included \\
\hline Multi-tiered & Yes & Hisex White & 36 & 75 & 18 \\
\hline Multi-tiered & Yes & Hisex White & 38 & 73 & 19 \\
\hline Multi-tiered & Yes & Lohmann LSL & 36 & 77 & 20 \\
\hline Single-tiered & No & Hisex White & 34 & 66 & 16 \\
\hline Single-tiered & No & Lohmann LB-Lite & 30 & $69^{1}$ & 20 \\
\hline Single-tiered & No & Lohmann LB-Lite & 32 & 75 & 17 \\
\hline Single-tiered & No & Lohmann LB-Lite & 36 & 72 & 11 \\
\hline Single-tiered & No & Lohmann LSL & 34 & 72 & 21 \\
\hline Single-tiered & Yes & Hisex White & 31 & 66 & 17 \\
\hline Single-tiered & Yes & Lohmann LSL & 34 & $62^{1}$ & 15 \\
\hline Single-tiered & Yes & Lohmann LSL & 34 & 74 & 20 \\
\hline Single-tiered & Yes & Lohmann LSL & 34 & 74 & 20 \\
\hline
\end{tabular}

In total, 214 hens were included.

${ }^{1}$ Hens in this flock were culled later than 1 week after the visit.

Table 2 Description of the scores used for the different welfare indicators

\begin{tabular}{|c|c|c|c|c|}
\hline \multirow[b]{2}{*}{ Condition } & \multicolumn{4}{|c|}{ Score } \\
\hline & 1 & 2 & 3 & 4 \\
\hline $\begin{array}{l}\text { Plumage: back (including rump), } \\
\text { belly (including vent), neck }\end{array}$ & $\begin{array}{l}\text { Very high graded damage of feathers with no } \\
\text { or very few feather covered areas. } \\
\text { Featherless areas }>5 \mathrm{~cm}^{2} \text { and almost bare } \\
\text { ( } 75 \% \text { to } 100 \% \text { featherless) }\end{array}$ & $\begin{array}{l}\text { Highly damaged feathers and/ } \\
\text { or featherless areas. } \\
\text { Featherless areas } \geqslant 5 \mathrm{~cm}^{2} \\
\text { (up to } 75 \% \text { featherless) }\end{array}$ & $\begin{array}{l}\text { Completely or almost } \\
\text { completely feathered; } \\
\text { few feathers damaged. } \\
\text { Featherless areas }<5 \mathrm{~cm}^{2}\end{array}$ & $\begin{array}{l}\text { Very good plumage; no } \\
\text { or very few feathers } \\
\text { damaged }\end{array}$ \\
\hline Plumage: tail & $\begin{array}{l}>13 \text { tail feathers highly damaged and/or } \\
\quad \text { almost bare }\end{array}$ & $\begin{array}{l}6 \text { to } 10 \text { tail feathers highly } \\
\text { damaged }\end{array}$ & $\begin{array}{l}6 \text { to } 10 \text { tail feathers } \\
\text { damaged }\end{array}$ & $\begin{array}{l}\text { Very good plumage; no } \\
\text { or very few feathers } \\
\text { damaged; } \leqslant 5 \text { tail } \\
\text { feathers damaged }\end{array}$ \\
\hline $\begin{array}{l}\text { Wounds: back (including rump) } \\
\text { and belly (including vent) }\end{array}$ & $>2.2 \mathrm{~cm}$ & $0.5 \geqslant \leqslant 2.2 \mathrm{~cm}$ & $<0.5 \mathrm{~cm}$ & Absent \\
\hline Keel bone deviation & $>1 \mathrm{~cm}$ & $0.5 \geqslant \leqslant 1 \mathrm{~cm}$ & $\leqslant 0.5 \mathrm{~cm}$ & - \\
\hline Keel bone fracture & Present & Absent & - & - \\
\hline Foot pad lesions & $>0.2 \mathrm{~cm}$ & $\leqslant 0.2 \mathrm{~cm}$ & Absent & - \\
\hline Plantar abscess (bumble foot) & Present & Absent & - & - \\
\hline Hyperkeratosis (foot pads) & Present & Absent & - & - \\
\hline Toe wounds & Present & Absent & - & - \\
\hline Missing toes/claws & Present & Absent & - & - \\
\hline Comb colour & Pale & Red (normal) & - & - \\
\hline
\end{tabular}

Modified from the HealthyHens scoring protocol.

same two trained observers working together on each hen and included scoring of plumage, keel bone, skin, feet and comb colour of each hen. The keel bone damages were examined by palpation, performed by running two fingers down each side of the keel bone and both vertical and horizontal deviations were scored. At the postmortem examination, faecal samples were collected from the cloaca after thawing, and a faecal egg count was obtained using the concentrate McMaster technique (Permin and Hansen, 1998) with a sensitivity of 20 eggs per gram (EPG) faces. A pilot study on two farms had revealed that the prevalence of infected hens diagnosed from fresh and frozen faecal samples, respectively, only differed with 7 to 9 percentage points (incidences were above $50 \%$ ) for what reason it was decided that it was acceptable to use frozen faecal samples. The faecal samples were weighed and the amount of flotation fluid ( $500 \mathrm{~g}$ glucose monohydrate $/ 1000 \mathrm{ml}$ saturated $\mathrm{NaCl}$ solution, specific 138 gravity of $1.27 \mathrm{~g} / \mathrm{ml}$ ) added to the sample was adjusted according to the amount of faecal material $(1.0 \mathrm{~g}$ of faecal material and $14 \mathrm{ml}$ flotation fluid). All eggs in the samples were identified using morphology, but $A$. galli and Heterakis sp. eggs have a similar appearance and size and were therefore not separated. Faecal egg count is the detection method normally used in relation to on-farm management of helminth infection under Danish conditions, which was why the decision was made to use the EPG levels for the classification of infection status. To investigate the correlation between faecal egg count and worm count, we also investigated the intestines in all hens for the number of $A$. galli and Heterakis sp. worms (both the subadult and adult stages). This was done by opening the intestines along their entire length with a pair of scissors. For $A$. galli the content of the small intestine was spread on a tray and the mucosa was washed gently with tap water to 
release worms, whereas for Heterakis sp. the content of caeca was spread out, washed with tap water and examined. The worms were identified according to McDougald (2013) and by using a protocol with morphological characteristics of intestinal helminths developed for the HealthyHens project. Heterakis sp. was recorded as present/absent, while the number of $A$. galli was counted. Hens from five of the 12 farms were dewormed during the study by use of flubendazol (either Verminator ${ }^{\circledR}$ or Flubenol ${ }^{\circledR}$, Elanco Animal Health $A / S$, Herlev, Denmark) at the recommended dose; three farms devormed one time, one farm twice and one farm three times. The deworming was done between 11 and 40 weeks before the end of lay visits.

\section{Data management}

Body feathering, that is the summed plumage scores for tail, neck and belly, was assessed according to the median score for the peak of lay and the end of lay visits, respectively. For the peak of lay visit, the median score was 12 (equal to the best score for all three body parts) implying that a score of 12 defined a good body feathering at the peak of lay. At the end of lay, the median score was 9 implying that a score $\geqslant 9$ defined a good body feathering at the end of lay. At the end of lay, the median score was equal to the criteria for poor plumage used by Tauson et al. (2005). Back feathering, that is plumage condition at the back, was assessed as poor if the score was $\leqslant 2$ and good if the score was $\geqslant 3$ based on the definition by Tauson et al. (2005).

With regard to the keel bone condition each hen was classified as either having keel bone damages, that is the hens had fractures and/or deviations, or as having a normal keel bone, that is the hens had no deviations or fractures. In the analysis on foot pad lesions data on presence of small and large lesions were pooled. A similar procedure was used for wounds on the back and belly.

Regarding the analysis of helminth infection, each hen was classified as infected with $A$. galli when the hen had an EPG value over 100 or otherwise as non-infected with A. galli as suggested by Heckendorn et al. (2009). Due to very few hens found infected with Heterakis sp. in the worm count, it was assumed that the EPG value was mainly due to presence of $A$. galli. The number of faecal samples collected during the peak of lay visit from the tagged hens relocated at the end of lay was too few for analysis to be conducted. Deworming was not taken into account in the analysis, because the treated flocks received deworming $>11$ weeks before the end of lay visit; a previous study has shown that the parasite egg output from $A$. galli in the faeces typically reappear 2 to 4 weeks after deworming (Höglund and Jansson, 2011).

\section{Statistical analysis}

The differences between prevalence of the clinical welfare indicators at the peak of lay and the end of lay visits were analysed using the Fisher's exact test. The associations between clinical welfare indicators with at least a $5 \%$ prevalence at the peak of lay and the end of lay, occurrence of
A. galli infection, age at the end of lay and housing system were studied using graphical models (Whittaker, 1990; Lauritzen, 1996; Labouriau and Amorim, 2008). A binary presentation (presence/absence of an indicator) of the parameters included in the model was chosen to keep the graphical model as simple as possible.

In graphical models, the variables examined are represented by vertices in a graph, that is a collection of vertices (points) and edges connecting the vertices (lines). Two vertices (i.e. two variables) are connected by an edge (line) when the conditional correlation between them, given all the other variables, is different from zero. The absence of an edge joining two variables indicates that the two variables are not significantly correlated given the other variables. According to the theory of graphical models (Whittaker, 1990; Jørgensen and Labouriau, 2012), two variables are directly connected in the graph if, and only if, they carry information on each other that is not already contained in the other variables included in the model. If two variables are only connected indirectly in the graph, that is two variables that are included in the graphical model but not directly connected by an edge, then the variables may be correlated, but this correlation would be spurious in the sense that it could be completely explained by a cascade of correlations with the other variables. The graphical model representing the joint distribution of the selected welfare indicators and the helminth infection (A. galli and Heterakis sp.) was inferred by finding the graphical model that minimises the Bayesian information criterion as implemented in the $\mathrm{R}$ package gRapHD (Abreu et al., 2010). This inference procedure has optimal properties (see Haughton, 1988). Confirmatory analyses were performed using a Monte Carlo version of the Fisher's exact test with 1000000 Monte Carlo samples.

\section{Results}

Of the originally 1200 tagged hens, $17.8 \%$ were assessed both at the peak of lay and at the end of lay. An increased prevalence of poor plumage and keel bone damages was found at the end of lay compared with the peak of lay (Table 3; $P<0.001$ ). In contrast, the occurrences of plantar abscess (bumble foot) and pale combs decreased significantly between the two visits (Table 3; $P<0.05$ ).

Regarding the helminth infection, the worm count showed that the hens classified as infected by the faecal egg count were all positive for $A$. galli, and in addition three of them also had a Heterakis sp. infection. Furthermore, $25.7 \%$ of the hens were infected with Emeria sp. and $12.6 \%$ with Capillaria sp. A total of 15 hens (7\%) classified as non-infected by the faecal egg count had worms present in the intestines. The faecal egg count was correlated with the worm count of $A$. galli (Spearman rank correlation coefficient; $r=0.73$ ).

Figure 1 shows the representation of the graphical model describing the associations between keel bone damages, 
Welfare indicators in organic layers

Table 3 Prevalence of clinical welfare indicators among the 214 examined hens at the peak of lay and the end of lay (number of hens in brackets), prevalence of Ascaridia galli infection and the level of significance of the difference between the two visits

\begin{tabular}{lccc}
\hline \hline & Peak of lay & End of lay & Fisher's exact test \\
\hline Back feathering (poor) $^{1}$ & $7.9 \%(17)$ & $23.4 \%(50)$ & $* * *$ \\
Body feathering (neck, tail, belly) (poor) $^{2}$ & $25.7 \%(55)$ & $43.5 \%(93)$ & $* * *$ \\
Keel bone fractures (present) & $3.7 \%(8)$ & $18.7 \%(40)$ & $* * *$ \\
Keel bone deviation (present) & $8.9 \%(19)$ & $26.6 \%(57)$ & $* * *$ \\
Keel bone damages (present) & $10.7 \%(23)$ & $29.0 \%(62)$ & $* *$ \\
Foot pad lesions (present) & $0.5 \%(1)$ & $0.5 \%(1)$ & ns \\
Plantar abscess (bumble foot) (present) & $3.7 \%(8)$ & $0.5 \%(1)$ & $\mathrm{ns}$ \\
Foot pad, hyperkeratosis (present) & $0.0 \%(0)$ & $0.5 \%(1)$ & $\mathrm{ns}$ \\
Toe wounds (present) & $0.5 \%(1)$ & $0.0 \%(0)$ & $\mathrm{ns}$ \\
Missing toes (present) & $1.4 \%(3)$ & $1.9 \%(4)$ & $*$ \\
Skin wounds (present) & $3.3 \%(7)$ & $0.9 \%(2)$ & - \\
Comb colour (pale) & $4.2 \%(9)$ & $0.5 \%(1)$ & - \\
A. galli (>100 EPG) & - & $56.1 \%(120)$ & \\
\hline \hline
\end{tabular}

EPG = eggs per gram.

${ }^{1}$ Poor plumage condition at the back was equal to a score $\leqslant 2$.

${ }^{2}$ Poor plumage condition at the peak of lay: score $\leqslant 11$ and at end of lay: score $\leqslant 8$.

${ }^{3}$ Total prevalence of keel bone deviation and/or fractures.

ns $P>0.05,{ }^{*} P<0.05,{ }^{* *} P<0.001$

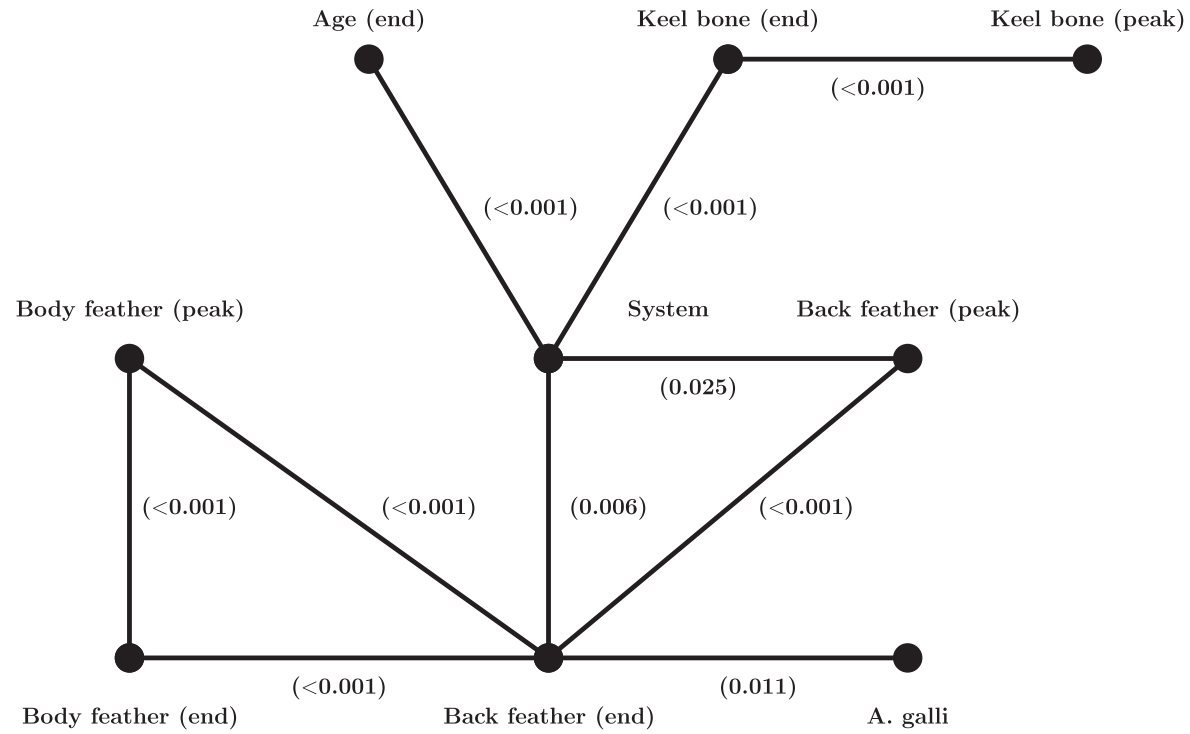

Figure 1 Representation of the graphical model showing the conditional correlations (edge (line) between two vertices (points representing the variables)) between the variables back feathering (Back feather), body feathering (Body feather) and keel bone damages (Keel bone) at the peak of lay (peak) and the end of lay (end), incidence of Ascaridia galli infection at the end of lay (A. galli), age at the end of lay (Age) and housing system (System). Hens were determined as infected or non-infected based on faecal egg count, with infected hens having an EPG over 100 and non-infected an EPG under 100. The level of significance for the direct association between two variables is presented in brackets below or beside the line. EPG = eggs per gram.

back feathering, body feathering, housing condition, age and A. galli infection. The only variable found to be directly connected to $A$. galli infection was back feathering at the end of lay. Thus, A. galli infection and back feathering at the end of lay were conditionally correlated given the other variables and, moreover, none of the other variables were conditionally correlated to $A$. galli infection. This indicates that all the information that the other welfare indicators may carry on $A$. galli infection was already contained in the variable back feathering at the end of lay. A Fisher exact test indicated that $A$. galli infection and back feathering at the end of lay were directly associated (see Figure $1 ; P=0.011$ ). The direction of this association was that $61.6 \%$ of hens with good plumage condition on the back were infected with A. galli, and $38.0 \%$ of hens with poor plumage condition on the back were infected with $A$. galli. Furthermore, as shown in Figure 1, back feathering at the end of lay was directly connected to housing system, back feathering at the peak of lay and body feathering at both the peak and end of lay. Poor feather condition at the back at the peak of lay and at the body at the peak or end of lay increased the risk of a poor back feathering at the end of lay. The housing system was 
directly connected to age, keel bone damages at the end of lay and to back feathering at the peak and end of lay. Hens from multi-tiered systems had a higher prevalence of keel bone damages than hens from single-tiered systems $(52.6 \%$ and $20.4 \%$, respectively). In addition, hens from multi-tiered systems had a lower prevalence of poor plumage condition on the back compared with hens from single-tiered systems both at the peak of lay $(0 \% \mathrm{v} .10 .8 \%)$ and at the end of lay $(15.8 \%$ v. $26.1 \%)$. Further, the end of lay visit was conducted at a higher age in multi-tiered systems compared with singletiered systems (mean 75 v. 70 weeks of age). Age at end of lay was only directly associated with housing system.

The clinical welfare indicators assessed at the end of lay were all directly connected with the corresponding clinical welfare indicator at the peak of lay (Figure 1). Thus, each pair of variables, representing the same welfare indicator, carried information on each other that was not already contained in the other welfare indicators. Furthermore, housing system separated keel bone damages from the other welfare indicators, indicating that keel bone damages were conditionally independent of the other welfare indicators given the housing system.

\section{Discussion}

\section{Association between the welfare indicators}

Our results provided evidence of an association between incidence of $A$. galli infection and back feathering at the end of lay with an increased incidence of $A$. galli infection in hens with a good plumage condition on the back. Moreover, the association was retained even after controlling for the possible effects of the other measured variables. This implies that the other welfare indicators considered did not carry any information about $A$. galli infection that was not already contained in the variable back feathering at the end of lay. Thus, the association reported was not a mere artefact of a complex composition of effects of the other welfare indicators. Moreover, when discussing the association between the clinical welfare indicators and $A$. galli infection it suffices to consider the association between $A$. galli infection and back feathering condition at the end of lay.

Ramadan and Abou Znada (1991) reported that helminth infected broilers showed ruffled feathers, and Green et al. (2000) reported an increased risk of feather pecking in flocks infected with intestinal worms. Feather pecking may result in plumage damage (Bilcik and Keeling, 1999; Tauson et al., 2005). Thus, previous studies have also found an association between feather condition and helminth infection. A plausible explanation for the reported association between A. galli infection and back feathering is that both $A$. galli infection and back feathering are associated positively with use of the outdoor area. Indeed, Green et al. (2000) also found feather pecking to be increased in flocks with poor outdoor use. Similarly, other previous studies have shown that the higher number of hens observed outside during scan samples, the better plumage condition of the flock (Lambton et al., 2010; Bestman and Wagenaar, 2014). As the outdoor area is a potential source of helminth infections (Heckendorn et al., 2009), a higher use of the outdoor area may in addition to improvements of the plumage condition also induce a higher risk for helminth infection. If the reducing effect on feather pecking gained by a higher use of the outdoor area is greater than the increasing effect on feather pecking caused by helminth infection, the result may be that hens with a high use of the outdoor area have a higher incidence of both helminth infection and good back feathering. This is of course highly speculative, that is further investigation of the association between back feathering and the incidence of $A$. galli infection is required to separate cause and effect.

Housing system and age at the end of lay were associated with a higher end of lay age in multi-tiered systems compared with single-tiered systems. This does not provide evidence that the age at depopulation was significantly different in the two housing systems, as two single-tiered farms were visited earlier than the last week before depopulation. Nevertheless, the end of lay visit was conducted at a younger age in single-tiered systems. This may have influenced the prevalence of hens with a poor plumage condition and with keel bone damages, as the prevalence of these variables increases with increasing age of the hens (Drake et al., 2010; Richards et al., 2012). An increased incidence of keel bone damages was indeed found in multi-tiered systems in the present study, which may be related to the higher mean age at the end of lay in the multi-tiered systems compared with single-tiered systems. Supporting this suggestion, Bestman and Wagenaar (2014) found no relationship between keel bone damages and whether the housing system was single-tiered or multi-tiered. However, others have found differences between housing systems with increasing complexity instigating higher occurrence of keel bone damages (Wilkins et al., 2011; Riber and Hinrichsen, accepted 1). Our results showed that keel bone damages were related to a casual path not including the other welfare indicators, as housing system separates keel bone damages from the other variables.

Back feathering, both at the peak and end of lay, and housing system were associated; an increased deterioration of the plumage condition on the back was found in singletiered systems compared with multi-tiered systems. As the hens at the end of lay were younger in the single-tiered system, this association cannot be explained by an increased accumulation of the welfare problem with age. However, interpreting the effects of housing system in the present study should be done with caution as only three of the 12 farms involved had multi-tiered systems. Riber and Hinrichsen (accepted 2) found a tendency for 62 weeks old hens in multi-tiered barn and organic systems to have a poorer plumage than hens in single-tiered systems. In contrast, Häne et al. (2000) found no difference in the plumage condition between multi-tiered and single-tiered systems (with permanent access to littered area) for hens between 50 to 70 weeks of age. The discrepancy is likely to 
be due to differences in the conditions laying hens are kept under in the countries involved in the two studies (i.e. Denmark and Switzerland). In addition, changes in the design of production systems, management guidelines and in genetic composition of the strains used may potentially explain the different results between studies conducted 15 years apart.

\section{Development of welfare indicators with age}

All clinical welfare indicators at the end of lay were associated with the same clinical welfare indicators at the peak of lay. This suggests that a welfare problem may be caused by the same common causal mechanisms irrespective of age. Alternatively, a welfare problem may accumulate over time, and thereby the prevalence of the problem may increase with age. These two possibilities are not necessarily mutually exclusive in the sense that they may both be present for a given welfare indicator, and they are not exhaustive in the sense that other more complex situations may occur. An example of this is the association between the back feathering at the peak and at the end of lay. Back feathering at the end of lay separated back feathering at the peak of lay from the variables body feathering and $A$. galli infections. This indicates that the condition of the plumage on the back at the end of lay was not only a result of accumulation over time, but that other factors besides increasing age also affected the plumage condition.

Previous studies have reported an increased prevalence of welfare problems with increasing age of the hens. This is the case for keel bone damages (Fleming et al., 2004; Richards et al., 2012) and plumage condition (Nicol et al., 2006; Drake et al., 2010). Damage to the plumage of pullets has been reported as a predictor for plumage condition in the laying period (Drake et al., 2010). The present study provides evidence that the plumage condition at the peak of lay can be a predictor for plumage condition at the end of lay. Our results show that back feathering at the end of lay can be predicted by both body and back feathering at the peak of lay. The plumage condition was separated into back and body featherings as the cause of the plumage damage on the back and body (tail, belly and neck) is different. Plumage damage to the back is usually related to feather pecking (Tauson et al., 2005; Welfare Quality, 2009) and is often the first area to become denuded in flocks expressing feather pecking (Bilcik and Keeling, 1999). The belly is also one of the first areas to become denuded (Bilcik and Keeling, 1999). However, this is often due to abrasion and is frequently seen in highly productive hens (Kjaer and Sørensen, 2002; Welfare Quality, 2009). Abrasion can also cause plumage damage on the neck and tail feathers (Bilcik and Keeling, 1999; Welfare Quality, 2009).

The prevalence of hens with pale combs and plantar abscess (bumble feet) decreased between the peak of lay and the end of lay. However, these results should be interpreted with caution as the number of hens categorised with pale combs and plantar abscess was low both at the peak and the end of lay. In contrast, there was an increase in the prevalence of hens with keel bone damages as well as poor plumage condition on the body and back between the visits. This is in agreement with previous studies (Drake et al., 2010; Richards et al., 2012; Riber and Hinrichsen, accepted 1,2).

\section{Prevalence of welfare indicators}

Bestman and Wagenaar (2014) reported that 32\% of the examined hens in commercial organic flocks were in a poor plumage condition at 50 to 60 weeks of age, which is fewer than found in the present study. In the same study by Bestman and Wagenaar (2014), 21\% of the hens had keel bone damages, a level similar to the prevalence of keel bone damages found in the present study. Similarly, another study in Denmark recently reported low prevalence of keel bone damage compared with other European countries (e.g. Nicol et al., 2006; Wilkins et al., 2011), which is thought to be related to differences in limestone supply (Riber and Hinrichsen, accepted 1).

The prevalence of hens with $A$. galli infection was in accordance with a previous report (Heckendorn et al., 2009). Other studies report the percentages of the flocks in the study being infected (>80\%; Pennycott and Steel, 2001; Sherwin et al., 2013; Bestman and Wagenaar, 2014). Prevalence of infected flocks and prevalence of infected hens are likely to be related but not directly comparable, as flock prevalence is the percentage of flocks that are infected based on the infection status of each of the flocks, while the prevalence of hens is the percentage of infected hens of the total hens investigated irrespectively of flock of origin.

\section{Conclusion}

In conclusion, back feathering at the end of lay was found to provide information about the likelihood of helminth infection at end of lay. We speculate whether high use of the outdoor area may cause a higher incidence of both helminth infection and good back feathering. To elucidate this further investigation of the association between back feathering and the incidence of $A$. galli infection is required. Unfortunately, no associations were found between any of the clinical welfare indicators at the peak of lay and helminth infection at end of lay. Back feathering at the end of lay is not a useful indicator in daily on-farm management practices of helminth infection. Finally, this study presented evidence that the deterioration of the plumage condition with age is not only due to accumulation of damage over time.

\section{Acknowledgements}

The authors would like to thank the producers for participating in the project and Trine Poulsen as well as Henrik Krogh Andersen for technical assistance. The project was funded by Aarhus University, Fund for Organic Farming (Fonden for Økologisk Landbrug) and the CORE Organic II Funding Bodies within the FP7 ERA-Net project HealthyHens (Coordination of European Transnational Research in Organic Food and Farming systems, project no. 249667). 


\section{References}

Abreu GCG, Edwards D and Labouriau R 2010. High-dimensional graphical model search with the gRapHD R package. Journal of Statistical Software 37, 1-18.

Anonymous 2008. Commission Regulation (EC) No 889/2008 of 5 September 2008, laying down detailed rules for the implementation of Council Regulation (EC) No 834/2007 on organic production and labelling of organic products with regard to organic production, labelling and control. Official Journal of the European Union L 250, 1-84.

Anonymous 2015. Statistics Denmark. Retrieved April 21, 2015, from http:// www.statistikbanken.dk/statbank5a/default.asp?w=1920

Arnould C, Butterworth A and Knierim U 2009. Standardisation of clinical scoring in poultry. In Assessment of animal welfare measures for layers and broilers. In Welfare quality report no. 9. (ed. Forkman B and Keeling L), pp. 7-30. SLU Service/Reproenheten, Uppsala, Sweden.

Barnett $J \mathrm{~L}$ and Hemsworth PH 2009. Welfare monitoring schemes: using research to safeguard welfare of animals on the farm. Journal of Applied Animal Welfare Science 12, 114-131.

Bartussek H 2001. Animal needs index for laying hens ANI 35-L/2001 - laying hens. Bundesantalt für Alpenländishe Landwirtschaft, Gumpenstein, Austria.

Bestman M and Wagenaar JP 2014. Health and welfare in Dutch organic laying hens. Animals 4, 374-390.

Bilcik B and Keeling LJ 1999. Changes in feather condition in relation to feather pecking and aggressive behaviour in laying hens. British Poultry Science 40, 444-451.

CORE organic HealthyHens project 2014. HealthyHens, good health and welfare in organic laying hens depends on good management. Retrieved August 11, 2015, from http://www.Coreorganic2.Org/healthyhens

Drake KA, Donnelly CA and Dawkins MS 2010. Influence of rearing and lay risk factors on propensity for feather damage in laying hens. British Poultry Science 51, 725-733.

Fleming RH, McCormack HA, McTeir L and Whitehead CC 2004. Incidence pathology and prevention of keel bone damages in the laying hen. British Poultry Science 45, 320-330.

Freire R, Wilkins LJ, Short F and Nicol CJ 2003. Behaviour and welfare of individual laying hens in a non-cage system. British Poultry Science 44, 22-29. Green LE, Lewis K, Kimpton A and Nicol C 2000. Cross-sectional study of the prevalence of feather pecking in laying hens in alternative systems and its associations with management and disease. Veterinary Record 147, 233-238.

Häne M, Huber-Eicher B and Frohlich E 2000. Survey of laying hen husbandry in Switzerland. World's Poultry Science Journal 56, 21-31.

Haughton DMA 1988. On the choice of a model to fit data from an exponential family. Annals of Statistics 16, 342-355.

Heckendorn F, Häring DA, Amsler Z and Maurer V 2009. Do stocking rate and a simple run management practice influence the infection of laying hens with gastrointestinal helminths? Veterinary Parasitology 159, 60-68.

Hinrichsen LK, Vaarst M, Sørensen JT. Danish organic egg producers' perception and experiences related to mortality and endoparasite infections. In Hinrichsen LK 2015. Animal welfare in organic egg production - with emphasis in mortality and helminth infections. PhD thesis, Aarhus University, Foulum, Denmark.

Höglund $J$ and Jansson DS 2011. Infection dynamics of Ascaridia galli in non-caged laying hens. Veterinary Parasitology 180, 267-273.

Jørgensen B and Labouriau R 2012. Exponential families and theoretical inference. Springer, Monografías de Matemática, Rio de Janeiro, Brazil.

Kjaer JB and Sørensen P 2002. Feather pecking and cannibalism in free-range laying hens as affected by genotype, dietary level of methionine plus cystine, light intensity during rearing and age at first access to the range area. Applied Animal Behaviour Science 76, 21-39.

Labouriau R and Amorim A 2008. Comments on "An association between the kinship and fertility of human couples". Science 322, 1634.
Lambton SL, Knowles TG, Yorke C and Nicol CJ 2010. The risk factors affecting the development of gentle and severe feather pecking in loose housed laying hens. Applied Animal Behaviour Science 123, 32-42.

Lauritzen SL 1996. Graphical models. Oxford University Press, New York, NY, USA.

Maurer V, Amsler Z, Perler E and Heckendorn F 2009. Poultry litter as a source of gastrointestinal helminth infections. Veterinary Parasitology 161, 255-260.

McDougald LR 2013. Internal parasites. In: Diseases of poultry Swayne, DE, Glisson, JR, McDougald, LR, Nolan, LK, Suarez, DL and Nair, VL, pp. 1117-1146. Wiley-Blackwell.

Nicol CJ, Brown SN, Glen E, Pope SJ, Short FJ, Warriss PD, Zimmerman PH and Wilkins LJ 2006. Effects of stocking density, flock size and management on the welfare of laying hens in single-tier aviaries. British Poultry Science 47, 135-146.

Pennycott TW and Steel F 2001. Parasitic worms in commercial free-range poultry flocks in England and Wales. Veterinary Record 149, 428.

Permin A, Bisgaard M, Frandsen F, Pearman M, Kold J and Nansen P 1999. Prevalence of gastrointestinal helminths in different poultry production systems. British Poultry Science 40, 439-443.

Permin A and Hansen JW 1998. Epidemiology. Diagnosis and control of Poultry parasites. FAO Animal Health Manual No. 4. Food and Agriculture Organization of the United Nations, Rome, Italy.

Pickel T, Schrader L and Scholz B 2011. Pressure load on keel bone and foot pads in perching laying hens in relation to perch design. Poultry Science 90, 715-724. Ramadan HH and Abou Znada NY 1991. Some pathological and biochemical studies on experimental ascaridiasis in chickens. Nahrung 35, 71-84.

Riber $A B$, Hinrichsen LK. Keel bone damage and foot injuries in commercial laying hens in Denmark. Animal, Accepted 1.

Riber $A B$, Hinrichsen LK. Feather eating and its associations with plumage damage and feathers on the floor in commercial farms of laying hens. Accepted 2.

Richards GJ, Wilkins LJ, Knowles TG, Booth F, Toscano MJ, Nicol CJ and Brown SN 2012. Pop hole use by hens with different keel fracture status monitored throughout the laying period. Veterinary Record 170, 494.

Sandilands V, Moinard C and Sparks NHC 2009. Providing laying hens with perches: fulfilling behavioural needs but causing injury? British Poultry Science 50, 395-406.

Sherwin CM, Nasr MAF, Gale E, Petek M, Stafford K, Turp M and Coles GC 2013. Prevalence of nematode infection and faecal egg counts in free-range laying hens: relations to housing and husbandry. British Poultry Science 54, 12-23.

Stratmann A, Fröhlich EKF, Harlander-Matauschek A, Schrader L, Toscano MJ and Würbel $H$ 2015. Soft perches in an aviary system reduce incidence of keel bone damage in laying hens. PLoS One 10, e0122568.

Tauson R and Abrahamsson P 1994. Foot and skeletal disorders in laying hens. Effects of perch design, hybrid, housing system and stocking density. Acta Agricultura Scandinavica. Section A, Animal Science 44, 110-119.

Tauson R, Kjaer J, Maria G, Cepero R and Holm K 2005. Applied scoring of integument and health in laying hens. Animal Science Papers and Report 23, 153-159.

Welfare Quality 2009. Welfare quality assessment protocol for poultry. Welfare Quality Consortium, Lelystad, The Netherlands.

Whittaker J 1990. Graphical models in applied multivariate statistics. John Wiley \& Sons, Chichester, UK.

Wier M, Jensen KO, Andersen LM and Millock K 2008. The character of demand in mature organic food markets: Great Britain and Denmark compared. Food Policy 33, 406-421.

Wilkins LJ, McKinstry JL, Avery NC, Knowles TG, Brown SN, Tarlton J and Nicol CJ 2011. Influence of housing system and design on bone strength and keel bone fractures in laying hens. Veterinary Record 169, 414. 